\title{
SOLVING THE HAMILTON-JACOBI EQUATION FOR GENERAL RELATIVITY
}

\author{
J. Parry, ${ }^{1}$ D. S. Salopek ${ }^{1,2}$ and J. M. Stewart ${ }^{1}$ \\ ${ }^{1}$ University of Cambridge, Department of Applied Mathematics and Theoretical Physics \\ Silver Street, Cambridge, CB3 9EW, UK \\ ${ }^{2}$ Department of Physics, University of Alberta, Edmonton, Canada T6G 2J1
}

(February 4, 2008)

\begin{abstract}
We demonstrate a systematic method for solving the Hamilton-Jacobi equation for general relativity with the inclusion of matter fields. The generating functional is expanded in a series of spatial gradients. Each term is manifestly invariant under reparameterizations of the spatial coordinates ("gauge-invariant"). At each order we solve the Hamiltonian constraint using a conformal transformation of the 3-metric as well as a line integral in superspace. This gives a recursion relation for the generating functional which then may be solved to arbitrary order simply by functionally differentiating previous orders. At fourth order in spatial gradients, we demonstrate solutions for irrotational dust as well as for a scalar field. We explicitly evolve the 3-metric to the same order. This method can be used to derive the Zel'dovich approximation for general relativity.
\end{abstract}

DAMTP R93/22

\section{INTRODUCTION}

In applications to cosmology, Hamilton-Jacobi (HJ) theory plays a vital role in describing the gravitational field. It serves as a starting point for a semi-classical analysis used both in stochastic inflation [1], [2], [3], and in quantum cosmology 酒. Recently it has been shown how to apply HJ techniques in solving the classical Einstein's equations. For example, one may derive the Zel'dovich approximation describing the formation of pancake structures in a matterdominated Universe [5], 6].

The Hamilton-Jacobi equation for pure gravity was first derived by Peres [7]. He showed that the momentum constraint implies the generating functional or the phase of the semi-classical wavefunctional remains invariant under arbitrary reparameterizations of the spatial coordinates (diffeomorphism invariance). However he was not able to solve the HJ equation. We find that a crucial ingredient is the introduction of matter fields. We also capitalize on the diffeomorphism invariance of the generating functional.

In this paper we expand the generating functional in a series of spatial gradients. This approximation scheme has a long history dating back to Lifshitz and Khalatnikov [8], as well as Eardley, Liang and Sachs [9]. In a quantum context, Teitelboim [10] and Pilati [11] considered an analogous approach which they called the strong-coupling expansion. Accurate to first order in spatial gradients, Salopek and Bond [3] explicitly solved the Hamilton-Jacobi equation and the momentum constraint with scalar fields present. In an important generalization, Salopek and Stewart [12] added dust fields and then demonstrated solutions to second order. The principal goal of this paper is to give solutions to higher order.

New techniques yield a practical and systematic method of computing higher order terms. It proves convenient to employ a conformal transformation of the 3-metric to simplify the Hamiltonian constraint, which can then be integrated using a line integral in superspace. A comparison with an exact solution shows that in situations of interest for cosmology even the first few terms can be quite accurate.

We start in Sec. II by writing out the Hamilton-Jacobi equation for general relativity including two types of matter fields, a regular scalar field $\phi$, and a dust field $\chi$ describing a pressure-free fluid of irrotational massive particles, for example, cold-dark-matter. The spatial gradient expansion is introduced, and the Hamilton-Jacobi equation is expanded to all orders in spatial gradients. Solutions of this equation correct to zero and two spatial gradients are reviewed. A new technique involving a conformal transformation is introduced in Sec. III in order to find solutions to higher orders. A recursion relation is derived which enables one to compute solutions to any given order, and we explicitly give the fourth order terms. For a single dust field, we solve the evolution equation for the 3-metric in Sec. IV, and show that our solution recovers the Szekeres exact solution [13] to Einstein's equations. This is followed by some concluding remarks. (Units are chosen so that $c=8 \pi G=\hbar=1$. The sign conventions of Misner, Thorne and Wheeler [14] will be adopted throughout.) 


\section{THE HAMILTON-JACOBI EQUATION FOR GENERAL RELATIVITY}

In HJ theory the primary object of interest is the generating functional $\mathcal{S}$, which is the phase of the semi-classical wavefunctional $\Psi \sim e^{i \mathcal{S}}$. For general relativity, the HJ equation is simply the energy constraint with the momenta replaced by functional derivatives of the 3 -metric. In addition we must consider the momentum constraint, which demands the gauge invariance of $\mathcal{S}$.

In this section we write $\mathcal{S}$ as a series of terms grouped according to the number of spatial derivatives that they contain. The Hamilton-Jacobi equation is likewise written as a sum of such terms. We review solutions for $\mathcal{S}$ correct to zeroth order (no spatial gradients) and to second order (two spatial gradients) for a single dust field, and for a single scalar field.

The action for general relativity interacting with a scalar field $\phi$ and a dust field $\chi$ is

$$
\mathcal{I}=\int d^{4} x \sqrt{-g}\left(\frac{1}{2}{ }^{(4)} R-\frac{1}{2} g^{\mu \nu} \partial_{\mu} \phi \partial_{\nu} \phi-V(\phi, \chi)-\frac{1}{2 m} n\left(g^{\mu \nu} \partial_{\mu} \chi \partial_{\nu} \chi+m^{2}\right)\right),
$$

where ${ }^{(4)} R$ is the Ricci scalar of the space-time metric $g_{\mu \nu}, \phi$ is a scalar field and $\chi$ is a velocity potential for irrotational dust particles of rest mass $m$ (which is a universal constant). $V(\phi, \chi)$ is a potential for the matter fields. The rest number density $n \equiv n(t, x)$ is a Lagrange multiplier which ensures that the 4-velocity

$$
U^{\mu}=-g^{\mu \nu} \chi_{, \nu} / m
$$

satisfies $U^{\mu} U_{\mu}=-1$. $\chi$ may be rescaled to include $m$, and so from now on we set $m=1$.

In the ADM formalism the line element is written

$$
d s^{2}=\left(-N^{2}+\gamma^{i j} N_{i} N_{j}\right) d t^{2}+2 N_{i} d t d x^{i}+\gamma_{i j} d x^{i} d x^{j}
$$

where $N$ and $N_{i}$ are the lapse and shift functions respectively, and $\gamma_{i j}$ is the 3-metric. We can then rewrite the action in Hamiltonian form,

$$
\mathcal{I}=\int d^{4} x\left(\pi^{\Phi_{a}} \dot{\Phi}_{a}+\pi^{i j} \dot{\gamma}_{i j}-N \mathcal{H}-N^{i} \mathcal{H}_{i}\right)
$$

Here the $\pi^{i j}$ denote the momenta conjugate to $\gamma_{i j}$ and $\Phi_{a}=(\phi, \chi)$ is an assembly of the scalar and dust fields with corresponding conjugate momenta $\pi^{\Phi_{a}}$. We use the summation convention over the subscript $a=1,2$. Variations of the lapse and shift functions produce the Hamiltonian and momentum constraints:

$$
\begin{aligned}
\mathcal{H}= & \gamma^{-1 / 2} \pi^{i j} \pi^{k l}\left[2 \gamma_{i l} \gamma_{j k}-\gamma_{i j} \gamma_{k l}\right]+\sqrt{1+\gamma^{i j} \chi_{, i} \chi, j} \pi^{\chi}+\frac{1}{2} \gamma^{-1 / 2}\left(\pi^{\phi}\right)^{2}+\gamma^{1 / 2} V\left(\Phi_{a}\right) \\
& -\frac{1}{2} \gamma^{1 / 2} R+\frac{1}{2} \gamma^{1 / 2} \gamma^{i j} \phi_{, i} \phi_{, j}=0, \\
\mathcal{H}_{i}= & -2\left(\gamma_{i k} \pi^{k j}\right)_{, j}+\pi^{l k} \gamma_{l k, i}+\pi^{\Phi_{a}} \Phi_{a, i}=0,
\end{aligned}
$$

where $R$ is the Ricci scalar of the 3 -metric.

Variation of the action (2.4) with respect to the momenta give the evolution equations for the field variables

$$
\begin{gathered}
\left(\dot{\phi}-N^{i} \phi_{, i}\right) / N=\gamma^{-1 / 2} \pi^{\phi}, \\
\left(\dot{\chi}-N^{i} \chi_{, i}\right) / N=\sqrt{1+\chi_{, i} \chi^{, i}}, \\
\left(\dot{\gamma}_{i j}-N_{i \mid j}-N_{j \mid i}\right) / N=2 \gamma^{-1 / 2} \pi^{k l}\left(2 \gamma_{j k} \gamma_{i l}-\gamma_{i j} \gamma_{k l}\right),
\end{gathered}
$$

where | denotes covariant differentiation with respect to the 3-metric. Variations of the action (2.4) with respect to the field variables yield the evolution equations for the momenta. However, these remaining evolution equations are automatically satisfied provided that

$$
\pi^{i j}(x)=\frac{\delta \mathcal{S}}{\delta \gamma_{i j}(x)}, \quad \pi^{\Phi_{a}}(x)=\frac{\delta \mathcal{S}}{\delta \Phi_{a}(x)},
$$


satisfy the constraint equations (2.5) and provided the evolution equations (2.6) hold. In this interpretation the energy constraint (2.5a) is a self-contained equation for $\mathcal{S}$, whereas the momentum constraint (2.5b) demands that $\mathcal{S}$ be gauge-invariant. The lapse and shift functions do not enter the constraints. Loosely speaking, equations (2.6) imply that trajectories in superspace are orthogonal to the phase functional. (In a more general formulation, $\mathcal{S}$ actually generates a canonical transformation to new variables [12].)

In terms of the generating functional, the Hamiltonian and momentum constraints are

$$
\begin{aligned}
\mathcal{H}(x)= & \gamma^{-1 / 2} \frac{\delta \mathcal{S}}{\delta \gamma_{i j}(x)} \frac{\delta \mathcal{S}}{\delta \gamma_{k l}(x)}\left[2 \gamma_{i l}(x) \gamma_{j k}(x)-\gamma_{i j}(x) \gamma_{k l}(x)\right] \\
& +\frac{1}{2} \gamma^{-1 / 2}\left(\frac{\delta \mathcal{S}}{\delta \phi(x)}\right)^{2}+\sqrt{1+\gamma^{i j} \chi_{, i} \chi, j} \frac{\delta \mathcal{S}}{\delta \chi(x)}+\gamma^{1 / 2} V\left(\Phi_{a}(x)\right) \\
& -\frac{1}{2} \gamma^{1 / 2} R+\frac{1}{2} \gamma^{1 / 2} \gamma^{i j} \phi_{, i} \phi_{, j}=0 \\
\mathcal{H}_{i}(x)= & -2\left(\gamma_{i k} \frac{\delta \mathcal{S}}{\delta \gamma_{k j}(x)}\right)_{, j}+\frac{\delta \mathcal{S}}{\delta \gamma_{l k}(x)} \gamma_{l k, i}+\frac{\delta \mathcal{S}}{\delta \Phi_{a}(x)} \Phi_{a, i}=0 .
\end{aligned}
$$

Equation (2.8a) is now called the Hamilton-Jacobi equation.

\section{A. Spatial Gradient Expansion of the Generating Functional}

Our method will be to expand the generating functional

$$
\mathcal{S}=\mathcal{S}^{(0)}+\mathcal{S}^{(2)}+\mathcal{S}^{(4)}+\ldots,
$$

in a series of terms according to the number of spatial gradients they contain. As a result the Hamilton-Jacobi equation can be grouped into terms with an even number of spatial derivatives:

$$
\mathcal{H}=\mathcal{H}^{(0)}+\mathcal{H}^{(2)}+\mathcal{H}^{(4)}+\ldots .
$$

\section{Solution of zeroth order Hamiltonian}

The Hamilton-Jacobi equation of order zero is

$$
\begin{aligned}
\mathcal{H}^{(0)}= & \gamma^{-1 / 2}{\frac{\delta \mathcal{S}}{\delta \gamma_{i j}}}^{(0)}{\frac{\delta \mathcal{S}}{\delta \gamma_{k l}}}^{(0)}\left(2 \gamma_{j k} \gamma_{l i}-\gamma_{i j} \gamma_{k l}\right)+\frac{1}{2} \gamma^{-1 / 2}\left(\frac{\delta \mathcal{S}}{\delta \phi}^{(0)}\right)^{2} \\
& +\frac{\delta \mathcal{S}}{\delta \chi}^{(0)}+\gamma^{1 / 2} V\left(\Phi_{a}\right)=0 .
\end{aligned}
$$

The diffeomorphism invariance of the generating functional suggests a solution of the form

$$
\mathcal{S}^{(0)}=-2 \int d^{3} x \gamma^{1 / 2} H\left(\Phi_{a}\right) .
$$

Because the integral is over $d^{3} x \gamma^{1 / 2}$ the functional is invariant under coordinate transformations, and it clearly contains no spatial derivatives. The numerical factor -2 is chosen so that $H$ corresponds to the usual Hubble parameter in the long-wavelength approximation. The following condition must be satisfied in order that the zeroth energy constraint vanish:

$$
H^{2}=\frac{2}{3}\left(\frac{\partial H}{\partial \phi}\right)^{2}-\frac{2}{3} \frac{\partial H}{\partial \chi}+\frac{1}{3} V(\phi, \chi) .
$$

We now consider two solutions of this nonlinear equation. Firstly for a single dust field and vanishing potential it is trivial to show that 


$$
H(\chi)=\frac{2}{3 \chi}
$$

is a solution. Setting the shift function to zero, and choosing our time coordinate to be $\chi$ (i.e., the lapse function $N=1$ ), solution of the metric evolution equation (2.6c) gives

$$
\gamma_{i j}^{(1)}(\chi, x)=\chi^{4 / 3} k_{i j}(x)
$$

where $k_{i j}(x)$, the 'seed' metric, is an arbitrary function of the spatial variables. This is the standard result for a dust-dominated universe at long-wavelengths.

Secondly we consider a scalar field self-interacting with an exponential potential

$$
V(\phi)=V_{0} \exp \left(-\sqrt{\frac{2}{p}} \phi\right),
$$

where $p$ is a constant. For this case, one can find analytic solutions. The equation (2.13) has an attractor solution

$$
H(\phi)=\left[\frac{V_{0}}{3(1-1 / 3 p)}\right]^{1 / 2} \exp \left(-\frac{\phi}{\sqrt{2 p}}\right) .
$$

For more details see ref. [3].

\section{Solution of the second order Hamiltonian}

In this section we will give a brief review of the second order, which was treated in Salopek and Stewart [12].

The second order HJ equation is

$$
\begin{aligned}
\mathcal{H}^{(2)}= & 2 H \gamma_{i j} \frac{\delta \mathcal{S}^{(2)}}{\delta \gamma_{i j}}-2 \frac{\partial H}{\partial \phi} \frac{\delta \mathcal{S}^{(2)}}{\delta \phi}+\frac{\delta \mathcal{S}^{(2)}}{\delta \chi} \\
& +\frac{1}{2} \gamma^{i j} \chi_{, i} \chi, j \frac{\delta \mathcal{S}^{(0)}}{\delta \chi}-\frac{1}{2} \gamma^{1 / 2} R+\frac{1}{2} \gamma^{1 / 2} \gamma^{i j} \phi_{, i} \phi_{, j}=0 .
\end{aligned}
$$

Their method was to write an Ansatz for the functional $\mathcal{S}^{(2)}$ which contains all terms that are second order in spatial gradients and are diffeomorphism invariant. There are three types of terms $-R, \Phi_{a, i} \Phi^{a, i}$ and $\Delta \Phi_{a}$.

$$
\mathcal{S}^{(2)}=\int \gamma^{1 / 2} d^{3} x\left(J\left(\Phi_{a}\right) R+K^{a b}\left(\Phi_{c}\right) \Phi_{a \mid i} \Phi_{b}{ }^{\mid i}+L^{a}\left(\Phi_{b}\right) \Delta \Phi_{a}\right) .
$$

The last term may be integrated by parts, and by redefining

$$
K^{a b} \rightarrow K^{a b}-\frac{\partial L^{a}}{\partial \Phi_{b}}
$$

we may set $L^{a}=0$. Substitution of the Ansatz (2.19) into the Hamilton-Jacobi equation at this order $(2.18)$ yields three sets of coupled partial differential equations which then must be solved. The number of equations exceeds the number of unknowns. However, no contradiction arises because some are redundant for reasons which will be discussed in the next section.

For a single dust field there are two independent equations.

$$
\begin{aligned}
& H J+\frac{\partial J}{\partial \chi}=1 / 2, \\
& 2 H \frac{\partial J}{\partial \chi}+K=0 .
\end{aligned}
$$

Given the zeroth order solution (2.14), these can be solved to give 


$$
\mathcal{S}^{(2)}=\int d^{3} x \gamma^{1 / 2}\left\{\left(\frac{3}{10} \chi+D \chi^{-2 / 3}\right) R+\left(-\frac{2}{5} \chi^{-1}+\frac{8}{9} D \chi^{-8 / 3}\right) \gamma^{i j} \chi_{, i} \chi, j\right\} .
$$

The term proportional to the arbitrary integration constant $D$ will be refered to as the complementary functional.

In an analogous way, for a single scalar field with the zeroth order solution (2.17), we find (neglecting the complementary functional)

$$
\mathcal{S}^{(2)}=\int d^{3} x \gamma^{1 / 2} \frac{p}{2(p+1)} \frac{1}{H}\left(R-\gamma^{i j} \phi_{, i} \phi_{, j}\right)
$$

\section{SOLVING THE HIGHER ORDER HAMILTONIAN}

The Ansatz method that was used to solve the second order case can be generalized to higher orders, but it is extremely tedious because the number of terms increases dramatically. For example the fourth order case has 15 types of diffeomorphism invariant terms leading to 15 coupled sets of partial differential equations. Although some of the free functions can be set to zero, just as in Sec. II.B.2, there are still eight variables satisfying fifteen equations. It is difficult to proceed further along these lines, and so we adopt an alternative approach.

Using the expression for $\mathcal{S}^{(0)},(2.12)$, we write down the Hamiltonian of order $2 n$ :

$$
\mathcal{H}^{(2 n)}=\left\{2 H \gamma_{i j} \frac{\delta \mathcal{S}^{(2 n)}}{\delta \gamma_{i j}}-2 \frac{\partial H}{\partial \phi} \frac{\delta \mathcal{S}^{(2 n)}}{\delta \phi}+\frac{\delta \mathcal{S}^{(2 n)}}{\delta \chi}\right\}+\mathcal{R}^{(2 n)}=0 .
$$

Here the remainder term $\mathcal{R}^{(2 n)}$, which is independent of $\mathcal{S}^{(2 n)}$, contains contributions from all previous orders, and is assumed to be known:

$$
\begin{aligned}
\mathcal{R}^{(2 n)}= & \gamma^{-1 / 2} \sum_{p=1}^{n-1} \frac{\delta \mathcal{S}^{(2 p)}}{\delta \gamma_{i j}} \frac{\delta \mathcal{S}^{(2 n-2 p)}}{\delta \gamma_{k l}}\left(2 \gamma_{j k} \gamma_{l i}-\gamma_{i j} \gamma_{k l}\right)+\gamma^{-1 / 2} \sum_{p=1}^{n-1} \frac{1}{2} \frac{\delta \mathcal{S}^{(2 p)}}{\delta \phi} \frac{\delta \mathcal{S}^{(2 n-2 p)}}{\delta \phi} \\
& +\sum_{p=1}^{n}\left(\begin{array}{c}
\frac{1}{2} \\
p
\end{array}\right)\left(\gamma^{i j} \chi_{, i} \chi_{, j}\right)^{p} \frac{\delta \mathcal{S}^{(2 n-2 p)}}{\delta \chi}+\mathcal{V}^{(2 n)}
\end{aligned}
$$

where the superspace potential $\mathcal{V}^{(2 n)}$ is defined to be

$$
\mathcal{V}^{(2 n)}= \begin{cases}-\frac{1}{2} \gamma^{1 / 2} R+\frac{1}{2} \gamma^{1 / 2} \gamma^{i j} \phi_{, i} \phi_{, j} & \text { for } n=1, \\ 0 & \text { otherwise. }\end{cases}
$$

Although the Hamiltonian constraint of order $2 n$, (3.1), is basically a linear equation for $\mathcal{S}^{(2 n)}$ (unlike the zeroth order equation (2.11)), it is nevertheless not immediately apparent how to calculate $\mathcal{S}^{(2 n)}$ given the previous orders, partly because the term in braces in equation (3.1a) is complicated. In this section we will show, firstly for a single dust field, and then for a single scalar field, a technique which yields a recursion relation for the generating functional. Finally we indicate briefly how to extend the analysis to multiple fields.

\section{A. Single Dust Field}

To simplify the HJ equation (3.1) for a single dust field, we utilise a conformal transformation of the 3-metric to define a metric variable

$$
f_{i j}(x) \equiv \Omega^{-2}(\chi(x)) \gamma_{i j}(x)
$$

where $\Omega$ satisfies

$$
\frac{\partial \Omega}{\partial \chi}=H \Omega
$$

The HJ equation (3.1a) reduces to 


$$
\left.\frac{\delta \mathcal{S}^{(2 n)}}{\delta \chi}\right|_{f_{i j}}+\mathcal{R}^{(2 n)}=0
$$

We note immediately that there are some obvious solutions to the homogeneous version of this equation $\delta \mathcal{S}^{(2 n)} /\left.\delta \chi\right|_{f_{i j}}=0$ which correspond to the complementary functionals. Any functional of only the conformal metric $f_{i j}$ that has $(2 n)$ spatial gradients and is diffeomorphism invariant will satisfy this equation. For example, at the second order $\mathcal{S}^{(2)}=D \int d^{3} x f^{1 / 2} \tilde{R}$ is the complementary functional, where $\tilde{R}$ is the Ricci scalar of $f_{i j}$. This corresponds to the arbitrary part of (2.22).

\section{The second order revisited}

To show how to use this conformal transformation, we shall now return to the second order HJ equation with one dust field present,

$$
\left.\frac{\delta \mathcal{S}^{(2)}}{\delta \chi}\right|_{f_{i j}}=-\mathcal{R}^{(2)}=\frac{1}{2} \gamma^{1 / 2} R+\gamma^{1 / 2} \frac{\partial H}{\partial \chi} \gamma^{i j} \chi_{, i} \chi, j
$$

The right hand side of this may be expressed in terms of the conformal metric $f_{i j}$ using the standard rules for canonical transformations, for example,

$$
R_{j}^{i}=\Omega^{-2} \tilde{R}_{j}^{i}+\Omega^{-1}\left(\Omega^{-1}\right)_{; j k} f^{i k}-\Omega^{-3} f^{k l} \Omega_{; k l} \delta_{j}^{i},
$$

where $R_{j}{ }_{j}$ is the Ricci tensor of the 3 -metric $\gamma_{i j}, \tilde{R}^{i}{ }_{j}$ is the Ricci tensor of $f_{i j}$, and a semicolon denotes covariant differentiation with respect to $f_{i j}$. Rewriting the right hand side and using the definition of the conformal factor (3.3), we find

$$
\left.\frac{\delta \mathcal{S}^{(2)}}{\delta \chi(x)}\right|_{f_{i j}}=-\mathcal{R}^{(2)}\left[\chi(x), f_{i j}(x)\right]=f^{1 / 2}\left(\frac{1}{2} \Omega \tilde{R}-f^{i j} \chi_{, i} \chi, j \frac{\partial}{\partial \chi}(H \Omega)-2 f^{i j} \chi_{; i j}(H \Omega)\right) .
$$

By inspection the solution is

$$
\mathcal{S}^{(2)}=\int d^{3} x f^{1 / 2}\left[j(\chi) \tilde{R}+k(\chi) \chi^{; i} \chi_{; i}\right],
$$

where the $\chi$-dependent coefficients $j$ and $k$ are

$$
j(\chi)=\int_{0}^{\chi} \frac{\Omega\left(\chi^{\prime}\right)}{2} d \chi^{\prime}+D, \quad k(\chi)=H \Omega,
$$

and $D$ is a constant. We can use the conformal transformation rules to find the generating functional in terms of the 3 -metric $\gamma_{i j}$ by using the zeroth order solution (2.14) and the conformal factor

$$
\Omega \equiv \chi^{2 / 3}
$$

which satisfies (3.3). This procedure recovers the generating functional (2.22) obtained by the direct approach. The constant of integration $D$ in the definition of $j$ yields the complementary functional at this order.

However, eq.(3.7) may be solved directly. A similar situation occurs in potential theory where one wishes to integrate an equation of the form

$$
\frac{\partial \phi}{\partial y_{j}}=g^{j}\left(y_{k}\right)
$$

which has the line-integral solution

$$
\phi\left(y_{k}\right)=\int_{C} \sum_{j} d y_{j}^{\prime} g^{j}\left(y_{l}^{\prime}\right)
$$


where $C$ is an arbitrary contour whose upper end point is $y_{k}$ and whose lower endpoint is arbitrary. If the two endpoints are fixed, the solution is independent of the choice of contour $C$ provided that $\sum g^{j} d y_{j}$ is exact. By analogy, if $\int d^{3} x d \chi(x) \mathcal{R}^{(2)}$ is exact, the solution of eq. 3.7) is

$$
\mathcal{S}^{(2)}=-\int_{C} \int d^{3} x d \chi^{\prime}(x) \mathcal{R}^{(2)}\left[\chi^{\prime}(x), f_{i j}(x)\right]
$$

The upper limit of integration is $\chi(x)$ and the lower limit $\chi_{0}(x)$ is an independent and arbitrary function. The complementary functional is set to zero by choosing $\chi_{0}=0$. We choose the simplest path for the line integral, that of a straight line in superspace:

$$
\chi^{\prime}(x)=s \chi(x), \quad d \chi^{\prime}(x)=\chi(x) d s, \quad 0 \leq s \leq 1 .
$$

The real parameter $s$ is analogous to the Tomonaga-Schwinger proper-time parameter [15]. The integration over $s$, $\int_{0}^{1} d s s^{2 / 3}=3 / 5$ is trivial leading to

$$
\mathcal{S}^{(2)}=-\int d^{3} x \frac{3}{5} \chi \mathcal{R}^{(2)}\left[\chi, f_{i j}\right]=\int d^{3} x f^{1 / 2} \frac{3}{5} \chi\left(\frac{1}{2} \Omega \tilde{R}-f^{i j} \chi_{, i} \chi, j \frac{\partial}{\partial \chi}(H \Omega)-2 f^{i j} \chi_{; i j}(H \Omega)\right),
$$

which, after an integration by parts, gives the same result as before, eq. 3.8.).

\section{The fourth order}

We illustrate the conformal transformation method for the fourth order. We rewrite $\mathcal{R}^{(4)}$ in terms of the conformal metric $f_{i j}$ and we find that, as before, we can integrate the equation directly: it is exact. The resulting generating functional written in terms of $f_{i j}$ is

$$
\mathcal{S}^{(4)}=\int d^{3} x f^{1 / 2}\left\{l(\chi)\left(\tilde{R}^{i j} \tilde{R}_{i j}-\frac{3}{8} \tilde{R}^{2}\right)+m(\chi) \tilde{R}^{2}+n(\chi)\left(\tilde{R}^{i j}-\frac{\tilde{R}}{2} f^{i j}\right) \chi_{; i} \chi_{; j}+r(\chi) \chi_{; i}{ }^{; i} \chi^{; j} \chi_{; j}\right\}
$$

where

$$
l(\chi)=-2 \int_{0}^{\chi} d \chi^{\prime} \frac{j^{2}\left(\chi^{\prime}\right)}{\Omega^{3}\left(\chi^{\prime}\right)}+L, \quad m(\chi)=M, \quad n(\chi)=-\frac{j(\chi)}{\Omega^{2}(\chi)}, \quad r(\chi)=-\frac{1}{4 \Omega(\chi)} .
$$

The two arbitrary constants, $L$ and $M$, give the additional complementary functional. Moreover, the complementary functional from the second order gives a contribution to this order through the dependence on $j$. This is a very succinct and tidy expression, which becomes more complicated when expressed in terms of the original 3-metric $\gamma_{i j}$, (using the conformal factor (3.9))

$$
\begin{aligned}
\mathcal{S}^{(4)}\left[\gamma_{i j}(x), \chi(x)\right]= & -\frac{3}{14} \int d^{3} x \gamma^{1 / 2}\left[\frac{9}{25} \chi^{3}\left(R^{i j} R_{i j}-\frac{3}{8} R^{2}\right)-\frac{3}{50} \chi R \chi_{\mid i} \chi^{\mid i}\right. \\
& \left.+\frac{3}{25} \chi R^{i j} \chi_{\mid i} \chi_{\mid j}+\frac{19}{50} \chi^{\mid i} \chi_{\mid i} \chi_{\mid j}^{\mid j}-\frac{1}{3 \chi} \chi^{\mid i} \chi^{\mid j} \chi_{\mid i} \chi_{\mid j}\right],
\end{aligned}
$$

where we have neglected all the complementary functionals.

\section{The recursion relation}

If for simplicity we choose to ignore all the complementary functionals at each order, we find that a simple recursion relation may be derived which relates each order to the previous orders, assuming that the remainder at each order is exact. It is trivial but tedious to show that the remainders $\mathcal{R}^{(2)}$ and $\mathcal{R}^{(4)}$ are exact by functionally differentiating $\mathcal{S}^{(2)},(3.8)$, and $\mathcal{S}^{(4)},(3.15)$. A general proof of the exactness of the remainder $\mathcal{R}^{(2 n)}$ and, as a result, the arbitrariness of the contour $C$ in the integral

$$
\mathcal{S}^{(2 n)}=-\int_{C} \int d^{3} x d \chi^{\prime}(x) \mathcal{R}^{(2 n)}\left[\chi^{\prime}(x), f_{i j}(x)\right],
$$


would rely on the fact that the Poisson bracket $\{\mathcal{H}(x), \mathcal{H}(y)\}$ yields the momentum constraint: integrability is assured provided gauge-invariance is maintained at lower orders. (See also DeWitt [16] and Moncrief with Teitelboim [17].)

By induction, one can show that each term in $\mathcal{R}^{(2 n)}$ on substitution of the line integral (3.13) has an $s$-dependence of $s^{2 n / 3}$. Hence the integral of the HJ equation (3.17) is

$$
\mathcal{S}^{(2 n)}=-\int d^{3} x \int_{0}^{1} d s \chi(x) \mathcal{R}^{(2 n)}\left[s \chi(x), f_{i j}(x)\right]=-\frac{3}{2 n+3} \int d^{3} x \chi(x) \mathcal{R}^{(2 n)}\left[\chi(x), f_{i j}(x)\right],
$$

and we may rewrite this in terms of the 3 -metric $\gamma_{i j}$

$$
\begin{aligned}
\mathcal{S}^{(2 n)}= & -\frac{3}{2 n+3} \int d^{3} x \chi(x) \mathcal{R}^{(2 n)}\left[\chi(x), \gamma_{i j}(x)\right] \\
= & -\frac{3}{2 n+3} \int d^{3} x\left\{\gamma^{-1 / 2} \sum_{k=1}^{n-1} \chi \frac{\delta \mathcal{S}^{(2 k)}}{\delta \gamma_{i j}} \frac{\delta \mathcal{S}^{(2 n-2 k)}}{\delta \gamma_{k l}}\left(2 \gamma_{j k} \gamma_{l i}-\gamma_{i j} \gamma_{k l}\right)\right. \\
& \left.+\sum_{k=1}^{n} \chi\left(\begin{array}{c}
\frac{1}{2} \\
p
\end{array}\right)\left(\gamma^{i j} \chi_{, i} \chi, j\right)^{k} \frac{\delta \mathcal{S}^{(2 n-2 k)}}{\delta \chi}+\chi \mathcal{V}^{(2 n)}\right\} .
\end{aligned}
$$

This expression has no reference to the conformal metric $f_{i j}$, and hence is easy to use in practice. In summary, to find the generating functional at each order, one must functionally differentiate the previous orders, and substitute in the above recursion relation.

\section{B. One Scalar Field}

To simplify the HJ equation (3.1) for a single scalar field we use a similar conformal transformation to the dust field case plus a change of variables for the scalar field. Many of the equations are similar. Once again, $f_{i j}(x)$ is defined by

$$
\gamma_{i j}(x) \equiv \Omega^{2}(u) f_{i j}(x),
$$

where the new variable $u(\phi)$ (which at the zeroth order is a comoving, synchronous time variable) is defined through an integral

$$
u=\int \frac{d \phi}{-2 \frac{\partial H}{\partial \phi}} .
$$

The conformal factor satisfies the ordinary differential equation

$$
\frac{\partial \Omega}{\partial u}=H \Omega
$$

For a single scalar field, the resulting HJ equation (3.1a) has a form similar to that of dust:

$$
\left.\frac{\delta \mathcal{S}^{(2 n)}}{\delta u}\right|_{f_{i j}}+\mathcal{R}^{(2 n)}=0,
$$

which again reduces the system to a simple first order functional differential equation with one independent variable.

$\mathcal{R}^{(2 n)}$ must be rewritten in terms of $f_{i j}$ and $u$. At the second order we find that it is exact, and the HJ equation (3.23) has the solution

$$
\mathcal{S}^{(2)}=\int d^{3} x f^{1 / 2}\left[j(u) \tilde{R}+k(u) u^{; i} u_{; i}\right]
$$

where

$$
j(u)=\int_{0}^{u} \frac{\Omega\left(u^{\prime}\right)}{2} d u^{\prime}+D, \quad k(u)=H \Omega .
$$

This expression is remarkably similar to the dust field case (3.8). For the exponential potential considered previously (2.16) with the zeroth order solution (2.17), $u$ and the conformal factor are defined by 


$$
u=p / H, \quad \Omega \equiv u^{p}
$$

These results can be used to show that the generating functional above (3.24) agrees with our original approach (2.23).

The fourth order solution also has a very similar form to the dust case.

$$
\mathcal{S}^{(4)}=\int d^{3} x f^{1 / 2}\left\{l(u)\left(\tilde{R}^{i j} \tilde{R}_{i j}-\frac{3}{8} \tilde{R}^{2}\right)+m(u) \tilde{R}^{2}+n(u)\left(\tilde{R}^{i j}-\frac{\tilde{R}}{2} f^{i j}\right) u_{; i} u_{; j}+r(u) u_{; i}^{; i} u^{; j} u_{; j}\right\}
$$

where

$$
l(u)=-2 \int_{0}^{u} d u^{\prime} \frac{j^{2}\left(u^{\prime}\right)}{\Omega^{3}\left(u^{\prime}\right)}+L, \quad m(u)=-\int_{0}^{u} d u^{\prime} \frac{\left(\frac{\Omega}{2}-j H\right)^{2}}{8\left(\frac{\partial H}{\partial \phi}\left(u^{\prime}\right)\right)^{2} \Omega^{3}\left(u^{\prime}\right)}+M, \quad n(u)=-\frac{j}{\Omega^{2}}, \quad r(u)=-\frac{1}{4 \Omega} .
$$

The main difference between this solution and the dust case $(3.15)$ is that here the coefficient of the $\tilde{R}^{2}$ term depends on $u$. Expressed in terms of the 3-metric $\gamma_{i j}$ and $\phi$ this becomes quite complicated.

$$
\begin{aligned}
\mathcal{S}^{(4)}=2 \frac{p}{p-3}\left(\frac{p}{2(p+1)}\right)^{2} \int d^{3} x \gamma^{1 / 2} & \frac{1}{H^{3}(\phi)}\left\{R^{k l} R_{k l}+\left(-\frac{3}{8}+\frac{1}{8 p}\right) R^{2}+\frac{1}{\sqrt{2 p}} R \phi_{\mid i}{ }^{\mid i}\right. \\
& +\frac{3}{4}\left(1-\frac{1}{p}\right) R \phi^{\mid i} \phi_{\mid i}+\left(\frac{3}{2 p}-2\right) R^{i j} \phi_{\mid i} \phi_{\mid j}+\phi_{\mid i}{ }^{\mid i} \phi_{\mid j}{ }^{\mid j} \\
& \left.+\left(-1+\frac{3}{4 p}\right) \frac{1}{\sqrt{2 p}} \phi_{\mid i}{ }^{\mid i} \phi^{\mid j} \phi_{\mid j}+\left(\frac{5}{8}-\frac{7}{8 p}+\frac{3}{8 p^{2}}\right) \phi^{\mid i} \phi_{\mid i} \phi^{\mid j} \phi_{\mid j}\right\}
\end{aligned}
$$

The recursion relation for a scalar field may be calculated in a similar way to the dust case. The integral of the HJ equation (3.23) may be written down

$$
\mathcal{S}^{(2 n)}=-\int_{u_{0}}^{u} \int d^{3} x d u^{\prime}(x) \mathcal{R}^{(2 n)}\left[u^{\prime}(x), f_{i j}(x)\right]
$$

where $u_{0}$ is a constant function which we can set to zero (this sets the complementary functionals to zero). Once again, as in the dust case (Sec. III.A.3), the exactness of the second and fourth order remainders can be shown explicitly. The exactness of the higher orders would seem to follow from the Poisson brackets of the constraints. One can solve (3.28) using a line integral as before

$$
u^{\prime}(x)=s u(x), \quad d u^{\prime}(x)=u(x) d s, \quad 0 \leq s \leq 1 .
$$

By induction, one can show that each term of $\mathcal{R}^{(2 n)}$, on substitution of this line integral, has an $s$-dependence $s^{(2 n-2)+(3-2 n) p}$, and hence

$$
\begin{aligned}
& \mathcal{S}^{(2 n)}=-\frac{1}{(2 n-1)+(3-2 n) p} \int d^{3} x u(x) \mathcal{R}^{2 n}\left[u, f_{i j}\right] \\
&=-\frac{1}{(2 n-1)+(3-2 n) p} \int d^{3} x \frac{p}{H(\phi)}\left\{\gamma^{-1 / 2} \sum_{p=1}^{n-1} \frac{\delta \mathcal{S}^{(2 p)}}{\delta \gamma_{i j}} \frac{\delta \mathcal{S}^{(2 n-2 p)}}{\delta \gamma_{k l}}\left(2 \gamma_{j k} \gamma_{l i}-\gamma_{i j} \gamma_{k l}\right)\right. \\
&\left.+\gamma^{-1 / 2} \sum_{p=1}^{n-1} \frac{1}{2} \frac{\delta \mathcal{S}^{(2 p)}}{\delta \phi} \frac{\delta \mathcal{S}^{(2 n-2 p)}}{\delta \phi}+\mathcal{V}^{(2 n)}\right\} .
\end{aligned}
$$

Again, this expression has no explicit reference to the conformal metric, and is easy to use.

\section{Multiple Fields}

One can solve the Hamilton-Jacobi equations for multiple fields by employing the method of characteristics. The characteristic equations are 


$$
\begin{gathered}
\frac{\partial \gamma_{i j}}{\partial \chi}=2 H \gamma_{i j} \\
\frac{\partial \phi}{\partial \chi}=-2 \frac{\partial H}{\partial \phi}
\end{gathered}
$$

with solution

$$
\begin{gathered}
\gamma_{i j}=\Omega^{2}(\chi(x)) f_{i j}(x), \\
\phi=h(\chi(x)+q(x)),
\end{gathered}
$$

where the $f_{i j}(x)$ and the $q(x)$ are constants of integration along a characteristic, and

$$
\frac{\partial \Omega}{\partial \chi}=H \Omega
$$

In this way equation (3.1a) has only one independent field $\chi(x)$.

$$
\left.\frac{D \mathcal{S}^{(2 n)}}{D \chi(x)}\right|_{f_{i j}, q}=-\mathcal{R}^{(2 n)},
$$

where $D$ denotes functional differentiation along a characteristic. One of the characteristic equations (3.32a) can be seen to define a conformal transformation.

\section{THE EVOLUTION OF THE 3-METRIC}

Using an iteration scheme, we show how to solve the evolution equation (2.60) for the 3-metric. We give explicit solutions to fifth order for a homogeneous dust field. A comparison is made with the Szekeres exact solution of Einstein's equations. The Zel'dovich approximation is briefly discussed. Firstly we find it useful to define a restricted generating functional.

\section{A. The restricted generating functional}

At the end of the calculation, we are free to choose our time hypersurface. For a single dust field, a natural choice is a surface of uniform $\chi$, which corresponds to comoving synchronous gauge - the evolution equation for $\chi,(2.6 \mathrm{~b})$, shows that the lapse $N=1$. For now on, we will also choose the shift function $N^{i}=0$. by

We define the restricted generating functional, $\mathcal{S}\left[\gamma_{i j}(x) \mid \chi\right]$, in which the dust field is assumed to be homogeneous,

$$
\mathcal{S}\left[\gamma_{i j}(x) \mid \chi\right] \equiv \mathcal{S}\left[\gamma_{i j}(x), \chi(x)=\chi\right] .
$$

The rate of change of the restricted generating functional $\mathcal{S}\left[\gamma_{i j}(x) \mid \chi\right]$ is

$$
\frac{\partial \mathcal{S}\left[\gamma_{i j}(x) \mid \chi\right]}{\partial \chi}=\int d^{3} x\left[\frac{\delta \mathcal{S}\left[\gamma_{i j}(x), \chi(x)\right]}{\delta \chi(x)}\right]_{\chi(x)=\chi}
$$

in which case the Hamilton-Jacobi equation implies the integrated Hamilton-Jacobi equation for $\mathcal{S}\left[\gamma_{i j}(x) \mid \chi\right]$

$$
0=\frac{\partial \mathcal{S}}{\partial \chi}+\int d^{3} x\left\{\gamma^{-1 / 2} \frac{\delta \mathcal{S}}{\delta \gamma_{i j}(x)} \frac{\delta \mathcal{S}}{\delta \gamma_{k l}(x)}\left[2 \gamma_{j k}(x) \gamma_{i l}(x)-\gamma_{i j}(x) \gamma_{k l}(x)\right]-\frac{1}{2} \gamma^{1 / 2} R\right\}
$$

and the momentum constraint reduces to

$$
0=-2\left(\gamma_{i k} \frac{\delta \mathcal{S}}{\delta \gamma_{k j}(x)}\right)_{, j}+\frac{\delta \mathcal{S}}{\delta \gamma_{l k}(x)} \gamma_{l k, i}
$$


The restricted generating functional is much easier to calculate in practice. The gradient expansion and the conformal transformation trick work for eq. (4.3) as well. However, integrability questions are not important here because there is only one equation to solve which always possesses solutions.

We can either compute the restricted functional using the integrated HJ equation (4.3), or using the full functional (3.16) and setting $\chi_{, i}=0$ at the end of the calculation. Both methods give the same answer. The restricted generating functional to fourth order is easily seen from (3.16).

$$
\mathcal{S}^{(4)}\left[\gamma_{i j}(x) \mid \chi\right]=-\int d^{3} x \gamma^{1 / 2} \frac{27}{350} \chi^{3}\left(R^{i j} R_{i j}-\frac{3}{8} R^{2}\right)
$$

One can go even further and compute the sixth order functional for a single homogeneous dust field:

$$
\mathcal{S}^{(6)}\left[\gamma_{i j}(x) \mid \chi\right]=\int d^{3} x \gamma^{1 / 2} \frac{54}{35}\left\{-\frac{49}{64} R^{3}+\frac{29}{8} R R^{i j} R_{i j}-4 R^{i j} R_{i}^{k} R_{j k}+R^{i j} R_{i j \mid k}{ }^{\mid k}-\frac{3}{8} R R^{\mid k}{ }_{\mid k}\right\} .
$$

\section{B. The evolution of the 3-metric for dust}

One can use these results to compute the evolution equation for the three-metric correct to higher order in spatial gradients for a single dust field. We adopt the iteration scheme proposed in reference [12]. The evolution equation computed from the ADM action (2.6d) on substitution of the spatial gradient expansion of the generating functional, truncating at sixth order is

$$
\frac{\partial \gamma_{i j}}{\partial \chi}=2 \gamma^{-1 / 2}\left(2 \gamma_{j k} \gamma_{i l}-\gamma_{i j} \gamma_{k l}\right)\left(\frac{\delta \mathcal{S}^{(0)}}{\delta \gamma_{k l}}+\frac{\delta \mathcal{S}^{(2)}}{\delta \gamma_{k l}}+\frac{\delta \mathcal{S}^{(4)}}{\delta \gamma_{k l}}+\frac{\delta \mathcal{S}^{(6)}}{\delta \gamma_{k l}}\right)
$$

where we have set the shift $N_{i}$ to be zero. We choose our time coordinate to be $\chi$ which fixes the lapse function $N=1$.

Retaining only the leading term on the right-hand side gives the first order result for $\gamma^{(1)}(\chi, x)$, eq. 2.15$)$. Substituting this expression into the right-hand side, and retaining only third order terms, one finds

$$
\frac{\partial \gamma_{i j}^{(3)}}{\partial \chi}=2 H \gamma_{i j}^{(3)}+\frac{3}{10} \chi\left(\hat{R} k_{i j}-4 \hat{R}_{i j}\right)
$$

where $\hat{R}_{i j} \equiv R_{i j}\left(k_{l m}\right)$ and $\hat{R} \equiv R\left(k_{l m}\right)$ are the Ricci tensor and Ricci scalar, respectively, of the seed metric $k_{l m}$. The third-order expression for the 3 -metric is then:

$$
\gamma_{i j}^{(3)}(\chi, x)=\chi^{4 / 3} k_{i j}(x)+\frac{9}{20} \chi^{2}\left[\hat{R}(x) k_{i j}(x)-4 \hat{R}_{i j}(x)\right]
$$

One more iteration leads to the fifth order expression

$$
\begin{aligned}
\gamma_{i j}^{(5)}= & \chi^{4 / 3} k_{i j}+\frac{9}{20} \chi^{2}\left(\hat{R} k_{i j}-4 \hat{R}_{i j}\right)+\frac{81}{350} \chi^{8 / 3}\left(\left\{\frac{89}{32} \hat{R}^{2}+\frac{5}{8} \hat{R}_{; k}^{; k}-4 \hat{R}^{k l} \hat{R}_{k l}\right\} k_{i j}\right. \\
& \left.-10 \hat{R} \hat{R}_{i j}+\frac{5}{8} \hat{R}_{; i j}+17 \hat{R}_{i}^{n} \hat{R}_{j n}-\frac{5}{2} \hat{R}_{i j ; k} ; k\right)
\end{aligned}
$$

where a semi-colon denotes covariant differentiation with respect to $k_{i j}$. A similar iteration scheme could be applied equally well to the scalar field case.

\section{Comparison to an exact solution}

In this section we will compare our approximation scheme to an exact solution of Einstein's equations - the axisymmetric specialization of the Szekeres solution [13 for irrotational dust, which has a line element

$$
d s^{2}=-d t^{2}+a^{2}(t)\left[d x^{2}+d y^{2}+\left(d\left(x^{i}\right)-c(z) a(t)\right)^{2} d z^{2}\right]
$$


where $d\left(x^{i}\right)$ has the form

$$
d\left(x^{i}\right)=d_{0}(z)-\frac{5}{9} c(z)\left(x^{2}+y^{2}\right),
$$

and $d_{0}(z)$ and $c(z)$ are arbitrary functions of $z$.

We interpret our evolution equation (4.10) as evolving the seed metric $k_{i j}(x)$ which we choose to be

$$
k_{i j}\left(x^{i}\right)=\operatorname{diag}\left[1,1, d^{2}\left(x^{i}\right)\right],
$$

where $d\left(x^{i}\right)$ is given by (4.12). We can now evaluate all the terms on the right hand side of (4.10). It is worthwhile using a computer algebra program, particularly for the fourth order part. Even for a simple metric such as (4.13) the individual terms such as $R_{i j ; k} ; k$ are quite complicated, and so it is extraordinary that the end result is so simple. We find that

$$
\gamma_{i j}^{(5)}=\chi^{4 / 3} \operatorname{diag}\left[1,1,\left(d\left(x^{i}\right)-c(z) \chi^{2 / 3}\right)^{2}\right],
$$

which is precisely the Szekeres solution (4.11)!

We can use this to check that there is no seventh order contribution to the Szekeres solution from our method by finding the solution to the sixth order evolution equation. We find that with the seed metric (4.13) there is indeed no contribution to the metric from the sixth order, i.e., $\gamma_{i j}^{(7)}=\gamma_{i j}^{(5)}$.

For situations of interest for cosmology, we do not wish to assume such a special form for the seed metric. For this case, the expansion can be improved quite significantly if one notes that the the determinant of the 3-metric must be non-negative. However, one disturbing property about the third order expression (4.9) is that after a sufficient amount of time, the 3-metric may no longer be positive definate — not only does the expansion break down, it gives nonsensical results. One may remove this embarrassing problem by writing (4.9) as a 'square':

$$
\tilde{\gamma}_{i j}^{(3)}=\chi^{4 / 3}\left\{k_{i l}+\frac{9}{40} \chi^{2 / 3}\left[\hat{R} k_{i l}-4 \hat{R}_{i l}\right]\right\} k^{l m}\left\{k_{j m}+\frac{9}{40} \chi^{2 / 3}\left[\hat{R} k_{j m}-4 \hat{R}_{j m}\right]\right\} .
$$

Both eqs.(4.9) and (4.15) agree to third order, but the latter is the preferred form because it is non-negative. This improved result reproduces the Zel'dovich approximation. See ref. [6] and [19] for a discussion of higher order terms.

\section{CONCLUSIONS}

In this paper we have developed a systematic method for solving the Hamilton-Jacobi equation for general relativity using a spatial gradient expansion. We derived a recursion relation by which we can compute the generating functional at each order by summing various combinations of the functional derivatives of lower orders. The key ingredients are a conformal transformation of the 3-metric and a line integral in superspace.

Our formalism provides some deep insights into the structure of semiclassical superspace which now far exceeds investigations in homogeneous models. Superspace describes an ensemble of evolving universes, and its complexity strains the imagination. However, the gradient expansion allows one to separate superspace into an infinite but discrete number of manageable pieces which are relatively easy to understand.

Because the line integral in superspace (see eq.(3.12)) is independent of the choice of contour (provided the endpoints are fixed), we now begin to understand the invariance of the generating functional under different time-hypersurface choices. The exactness of the remainder terms is highly nontrivial, and this important property can essentially be traced to the fact that the Poisson bracket of the Hamiltonian constraints, $\{\mathcal{H}(x), \mathcal{H}(y)\}$, yields the momentum constraint. Integrability is closely related to the gauge-invariance of the theory.

The first few terms of the gradient expansion are of considerable interest for a dust-dominated Universe describing, for example, the cold-dark-matter model. It is quite reassuring that the fifth order 3-metric reproduces the exact Szekeres solution. In addition, HJ theory provides an efficient and practical means of calculating higher terms in the Zel'dovich approximation [6], 19]. However, we have not discussed the convergence of the expansion. For more general problems, it seems likely that one must employ techniques that will improve the rate of convergence [18]. 


\section{ACKNOWLEDGMENTS}

Both D.S.S. and J.P. were funded through the Science and Engineering Research Council of the U.K.. D.S.S. also received partial support from the Natural Sciences and Engineering Research Council of Canada, and a Canadian Institute for Theoretical Astrophysics National Fellowship held in Edmonton.

[1] A.A. Starobinsky, in Current Topics in Field Theory, Quantum Gravity, and Strings, ed. H T de Vega and N Sanchez (Springer: New York, 1986), p.107.

[2] J.M. Stewart, Class. Quantum Grav. 8, 909 (1991).

[3] D.S. Salopek and J.R. Bond, Phys. Rev. D 43, 1005 (1991); ibid. 423936 (1990); D.S. Salopek, ibid. 43, 3214 (1991).

[4] J.J. Halliwell and S.W. Hawking, Phys. Rev. D 31, 1777 (1985).

[5] Y.B. Zel'dovich, A \& A, 5, 84 (1970).

[6] K.M. Croudace, J. Parry, D.S. Salopek and J.M. Stewart, Ap. J. (in press, 1993).

[7] A. Peres, Nuovo Cim. 26, 53, (1962).

[8] E. M. Lifshitz and I.M. Khalatnikov, Sov. Phys.-Usp. 6, 495 (1964).

[9] D. Eardley, E. Liang and R. Sachs, J. Math. Phys. 13, 99 (1972).

[10] C. Teitelboim, Phys. Rev. D 25, 3159 (1982).

[11] M. Pilati, Phys. Rev. D 26, 2645 (1982).

[12] D.S. Salopek and J.M. Stewart, Class. Quantum Grav. 9, 1943 (1992).

[13] P. Szekeres, Commun. Math. Phys. 41, 55 (1975).

[14] C. Misner, K.S. Thorne and J.A. Wheeler, Gravitation (New York: Freeman, 1973).

[15] S. Tomonaga, in Quantum Electrodynamics, ed. J. Schwinger (Dover, New York, 1958); ibid. J. Schwinger.

[16] B.S. DeWitt, Phys. Rev. 160, 1113 (1967).

[17] V. Moncrief and C. Teitelboim, Phys. Rev. D 6, 966 (1972).

[18] D.S. Salopek, J.M. Stewart and J. Parry, Phys. Rev. D 48, 719 (1993).

[19] D.S. Salopek, J.M. Stewart, K.M. Croudace and J. Parry, in Proc. 37th Yamada Conference, June 8-12, 1993, Tokyo, Japan, ed. K. Sato (Universal Academic Press, Inc. 1994). 